\title{
Doctor on Net with Effective Medicine Dispatcher in Medical Analysis
}

\author{
Parthasarathy $\mathrm{G}^{\mathrm{a}, 1}$, Soumya T.R ${ }^{\mathrm{b}}$, Ramesh $\mathrm{P}^{\mathrm{c}}$, and L.Ramanathan ${ }^{\mathrm{d}}$ \\ ${ }^{a}$ School of Computing and IT, REVA University, Karnataka, India \\ ${ }^{b}$ Dept of CSE, Jeppiaar Maamallan Engineering College, India \\ ${ }^{c}$ Dept of EEE, CMR Institute of Technology, Bengaluru, India \\ ${ }^{d}$ School of Computer Science and Engineering, VIT University, India
}

\begin{abstract}
Tracking the good doctors for treatment in rural area is a very difficult job, to requiring the rural folk to track all the way to nearby towns or cities. Therefore, Anytime Medical Machine (AMM) requires deployment in rural areas using the AMM with the ability of people to get good doctors on track and take the treatment through the AMM. The doctor provides medicines on prescription for the patients through AMM. This collects details relating to the patient's Body Temperature, Heart Beat, Height and Weight using Pi Kit. The Patient desires to see a language known to both of them and sending the collected information to the doctor through AMM. Interaction between the doctor and the patient follows using video conferencing it attached with AMM. Finally, the Doctor observes the patient's sickness and then suggests the appropriate medicine through AMM. The patient's information is uploaded in the cloud server. Admin re-fills the medicines in AMM during the intake timing. This AMM application performance level of the data result proven that the efficiency of $90 \%$. The significance of AMM plays a main role in automation of medicine dispatcher.
\end{abstract}

Keywords. Internet Protocol, Real-time Transfer Protocol, SHA-256 and Wireless Fidelity

\section{Introduction}

Tracking an experienced doctor's in the ambience will economizes time and effort, websites are available to help contacting doctors online, but they are not of much use in getting appropriate treatment for the patient. Therefore patient information is collected using a microcontroller containing temperature sensor, heartbeat sensor, load cell sensor and ultrasonic sensor, while every information about the patient related to taking treatment the data will be stored in the machine storage, when person contacting doctor data will be shared to the doctor. For more interaction with realistic video conferencing is the best way, the video conferencing is achieved through using IP address and Wi-Fi Modules. Based on two machines IP address and the Wi-Fi make the path for the video conferencing and it is established. So far patient and doctor get interact with each other. After the conversation, ends the medicine for the patient is dispatched from the Anytime Medicine Machine (AMM) through contacted doctor, if the medicine are not available it will be are refilled in the machine. In future patient in taking the medicine the contacted doctor will provide the prescription for the patient through AMM. If the patient suffers, more due to the treatment the contacted doctor details and the patient major information are get stored in database

${ }^{1}$ Parthasarathy G, UG scholar ,School of Computing and IT, REVA University, Karnataka, India; Email:id: parthasarathy.g@reva.edu.in 
and cloud. Cloud Storage is mostly used in day-today life for storage of useful information, now days they are many types of Cloud in the industries mostly everyone prefers hybrid cloud and other services like private and public cloud, where public cloud will not be a secure one, because everyone can use it without any user security. The private cloud is secure one with user authentication. The Hybrid cloud is the combination of both services. Here we use private cloud for storing patient information in secured manner. Higher officials of health department view the patient's information relating to the patients are get suffered more by the contacted doctor's treatment.

\section{Materials and Methods}

People living in cities have the facility of a doctor or clinic in their short distance for consultation, taking the necessary tests and getting the prescription of the appropriate medicine. Such facilities are not available for persons in rural areas who have to track a long distance. The concomitant problems are: The process involves considerable time, effort and expenditure.

Disadvantage are below:

\subsection{Unreliable}

Patient going to the hospital and consulting the doctors, then taking all necessary test and doctor prescribe the medicine we want to buy a medicine form the medical shop.

\subsection{Time consuming}

The patient want to going to the hospital and taking the entire test, then buying the medicine from the shop will consume more time.

\subsection{No Systematic Concept}

In Existing System, they are no systematic concepts everyone wants to take the test by going diagnostic center. It is common knowledge that the health and wellness part is a critical area for people with the requirement of the use of advanced technologies like IOT. Some among the Internet of Medical Things (IoMT) connected with IoT networks support monitoring the day to day health illness of the patients. In recent times there has been tries to style new medical devices that are of use specifically for the elderly persons in monitoring their health parameters. One such device is the MEDIBOX which is of help in assisting patient to take the medicines at the right time. This one is a highly efficient system that deals with the health parameters that include temperature in a well-ordered range recommended by doctors and manufactures of drugs. Associated with this another system developed in the Host Management System (HMS) which has the special facility of cloud installation that helps modification of the existing facilities in the design aspects [1].

Among other systems that have emerged in recent years is an expert system that is of immense use in diagnosis and reducing the time required for treatment with the use of AODV protocols the vital and critical parameters of a patient evaluated by WBAN are forwarded on requisition. AODV procedure can be modified according to the patient's condition in the event of any emergency. There is also an improved event 
driven AODV (MED-AODV). This supports the doctor in devising appropriate steps for the immediate treatment for the patients. When a need for specialists is felt the clustered AODV (C-AODV) is initiated. Clustered AODV is of exclusive assistance for the patient in helping the doctor to provide the prescription inspection of his location [2].

Security and privacy for information relating to health care are becoming matters of great concern. Digital patient records, enhanced regulation, consolidation indicate the trend towards improved information security matters relating to these are subjects in articles in medical formulas and those relating to law and health information [3].Application of intelligent program with physiology signal monitoring for e-health care is assuming great importance currently. This can help doctors in the effective monitoring of human physiology and economizing in the time and cost involved in visiting doctors. The study employees a system on chip (SOC) techniques to help development of an embedded human pulse monitoring system with intelligent knowledge analysis mechanism for malady detection and long health care. This can help monitoring and analysis of pulse signals. It also implied friendly web-based interface that facilitates the observation of the immediate physiological signals. An intelligent data analysis scheme based on the modified cosine similarity assists diagnosis of abnormal pulses in the matter of chronic diseases. This has brought in special benefits in terms of assisting long-distance medical treatment, getting a clear understanding of potential chronic diseases [4].

Data duplication is the current data compression method that helps economizing data storage space through elimination of duplicate data stored and keeping a set of information. Digital data is raising fast as a output of growth in the usage of internet and IoT [Internet of Things] devices. Multiple users are dealing with the similar data. This helps generation of many copies of identical data and their storage as multiple entries, resulting in which outputs need large quantity of storage space requirements. Duplication helps storage of one physical mirror of the data and referencing all duplicate entries. This technique eliminates the need for the storage of multiple entries of the similar information and saves considerable space. It also produces good results than other text data compression techniques [5-6].In this paper the author designed prototype model for IoHT to cure four available neuromotor diseases it is one amyotrophic lateral sclerosis. The performance of this model is partitioned into seven steps [7]. In this article author proposed a new network model of automation of health monitoring to develop the Quality of Service. To describe the accurate system result with a set of numerical data are given in proposed system.

\section{Proposed Work}

Temperature sensor, an ultrasonic sensor and a Heartbeat and Load cell sensor. For collecting patient's information assists the doctor in the examination of the patient's health condition and for a useful interaction with the patient.Video conferencing is included; the doctor examines the patient using the interaction and prescribes medicine which is dispatched from the AMM machine along with the prescription. Patient's information is stored in the cloud. In event of the prescription being ineffective, a review can be made and details stored in the cloud to enable examination of further process needed and the advantages are listed below. 


\subsection{Reliability}

The patient consults the doctor after taking all the tests using the machine.

\subsection{Time Consumed}

Time Consumed is less compared to the existing model as everything is done by AMM.

\subsection{Systematic Concept}

This is a systematic concept not everyone want to go to the diagnostic center for certain test.

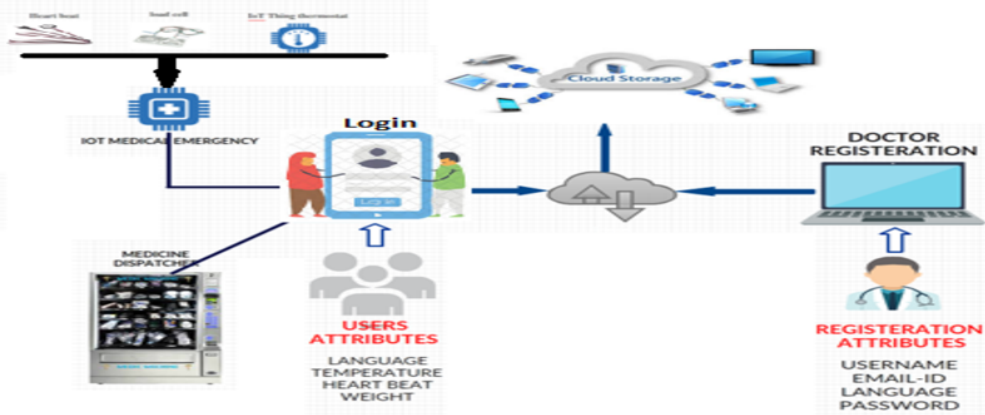

Figure 1. Architecture of Proposed System

The systematic concept where there is no need to go to the diagnostic center for tests. The proposed model is a highly useful diagram that uses available standards, wherein the primary work is to illustrate a specific set of alternatives inherent in the structure and design of a system or ecosystem. The architecture of the project from collecting the patient information through sensor, storing it and transferring it to the doctor through IP address using Wi-Fi module, storing it to the cloud, and dispatching the medicine are illustrated in Figure 1.

\subsection{Comparison}

\begin{tabular}{|l|l|}
\hline \multicolumn{1}{|c|}{ EXISTING SYSTEM } & \multicolumn{1}{c|}{ PROPOSED SYSTEM } \\
\hline $\begin{array}{l}\text { It is not reliable. The patient desires to track } \\
\text { good doctors for treatment. }\end{array}$ & $\begin{array}{l}\text { It is reliable. A doctor can be tracked } \\
\text { using AMM. }\end{array}$ \\
\hline $\begin{array}{l}\text { There is no proper method for monitoring } \\
\text { the treatment given to the patient by } \\
\text { the doctor. }\end{array}$ & $\begin{array}{l}\text { The complete patient information including the } \\
\text { treatment given to the patient is monitored } \\
\text { through the cloud. }\end{array}$ \\
\hline $\begin{array}{l}\text { This is not an efficient method for applying } \\
\text { first aid to an injured candidate. }\end{array}$ & $\begin{array}{l}\text { It is efficient method for to get first aid for an } \\
\text { injured candidate through AMM. }\end{array}$ \\
\hline $\begin{array}{l}\text { We take waiting for doctors for the treatment } \\
\text { is a time consuming process. }\end{array}$ & $\begin{array}{l}\text { It saves time for patient in taking treatment } \\
\text { waiting for doctors. }\end{array}$ \\
\hline
\end{tabular}




\subsection{Embedded Hardware Construction with user information}

User Information: User can access the application through entering test information taken by him/her connected with the AMM and selecting the language of patient familiar to him/her for establishing communication between doctors. Doctor wants to make use the application means they want to provide the personal vitae and interests are to be registered. Once created, the doctor attends to the patients call and gets logged from the application.

Heartbeat sensor: A heartbeat is that the pumping sound of the valves in individual heart during contracting expansion as the blood emerges How many amount of times the heart beats per minute (BPM), is the heart beat speed and the beat of the heart can be felt in artery that lie down neat to the skin is the pulse.

The LM35 will easily connect with new integrated circuit temperature sensors. It is fixed in a surface and the temperature will be at the intervals of the value may vary of $0.01^{\circ} \mathrm{C}$ of the surface temperature. This is based on the assumption of the ambient surface temperature being approximately will remains same; When the air temperature is higher or lower than surface temperature, then the actual temperature of the LM35 would be die the level of temperature between the surface temperature and the air temperature.

Temperature sensors found extensive applications in environmental and method management and conjointly in take a look at, measurement and communication. The digital temperature may be a detector that provides 9-bit temperature readings. Digital temperature sensors are known for excellent accuracy, therefore it is designed to read from $0^{\circ} \mathrm{C}$ to $70^{\circ} \mathrm{C}$ and the possibility of achieving $\pm 0.5^{\circ} \mathrm{C}$ accuracy. These types of sensors utterly aligned with digital temperature readings in degree Centigrade.

Ultrasonic Sensor: Ultrasonic discovering is the most ordinarily utilized device in industrial applications for the detection of hidden objects. For this purpose physics, which indicate the propagation of sound waves through solid materials have been used. Ultrasonic sensors is used sound instead of using the light for the detection. Sound that is mechanical wave movement in the mediums, whether it may be in any state. Sound waves will travel through the any mediums with specific rate. The sound waves that area unit having high frequency replicate from boundaries and produce unique echo patterns. Weight Sensor: A load cell is a device that converts a load or force engaged on it into associate in nursing sign. Which could be any one of this type like voltage, current or frequency depending on the type of which load cell we used Figure 2 is a load cell and circuitry used. Now they are varies kinds of load cells are used. Resistive load cells and electrical phenomenon load cells are offered.

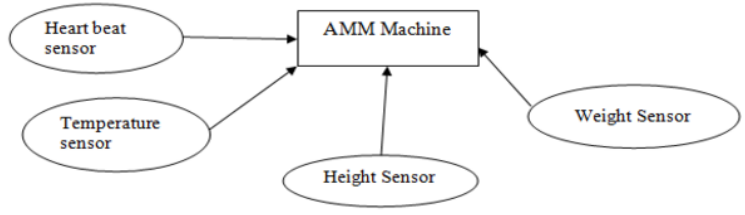

Figure 2. AMM Machine Database

A single point load cell that calculates the weight of the person where a single weight holds 10 kilograms is used. 


\subsection{Biomedical Analysis}

The bio medical analysis process[9] where implemented and designed in project. Once the patient has taken the test, the information gets collected and stored in the machine database . Patient consulting the doctor at that time the patient information is their shown to the doctor in a medicine availability screen video conferencing that follows enables in doctor and patient side in voice and video communication with doctor about patient health. The doctor indicates the treatment and prescribes the medicine for the patient. Finally, the patient information gets stored in the cloud storage. Through the use of API with user application and a secret key, where the data get encrypted while transforming to cloud. An authorized person for the cloud can view the patient information. This has the username and their test values.

\subsection{Medicine Dispatch}

The machine will dispatch the medicines are forwarded to the patients using the AMM machine. Where all the medicines details are updated within the storage and then the consulted doctors will dispatches the medicine and prescription from the medicine dispatcher to the patient. After consulting a doctor, they can send the request to the machine dispatcher for dispatching the medicine from the AMM, afterward only the patient get the tablets and using prescription then can get medicine in future. If the medicine is dispatched, the both doctor and patient make a call and then only doctor can able to consult another patient through video and voice communication.

\section{Results}

\subsection{Real-Time Transfer Protocol (RTP) Algorithm}

It is a network protocol where it is used in wired and wireless medium that provides the facility of transfer of audio and video using IP Address with other parties. It mainly for communication and entertainment. Involving streaming media that include telephony, video teleconference applications. RTP uses the User Datagram Protocol (UDP) for conjunction with the RTP Control Protocol (RTCP). It carries the media streams and RTCP is utilized for viewing the statistic transmission, quality of service and aids synchronization of multiple streams. RTP algorithm has been used by the author for video transmission between the doctor and the patient for useful interaction. Its function is based completely on IP Address of two machines.

\subsection{Secure Hash Algorithm (SHA)-256}

It is a type of hash function most probably using in block chain and API (Application Programming Interface) call. It is a like a mathematical function and it will turns data to hash[8]. It resembles a formula or algorithm. It will take the input data, and then turned it into the output for a fixed length, representing in the form of data SHA-256 has been used for the storage of the data in cloud storage through use of an API call. Encryption of API key is done using SHA-256, while calling; it gets decrypted in the application and the data is stored. 


\section{Discussion}

The patients can get treatment for pain easily with the help of AMM; the treatment includes medicine for use in emergency. Currently everything is seen as systematic format. So we implemented the doctor providing the treatment for the patient is done through use of a machine and a system. To start with, the patient takes all necessary tests using the sensors connected with a microcontroller fixed to AMM. It provides the real time feel of visiting a hospital to the patient. After the consulting, the doctor provides the medicine and the prescription of the medicine. Patient information gets updated in cloud in a secure manner. But the existing procedure will only be a barrier to the patients who have suffered pain and sickness. This reduces the patient's time, involved in waiting for consulting doctors. The Table 1 have checked 5 variety of patients by applying different sensors and we have measured temperature, pressure and heart beat pulse values. Every patients information is given in the Table 1 then the data visualization are given the figure 3 .

\section{Table 1. Patients Information}

\begin{tabular}{|l|c|c|l|l|l|l|l|l|l|}
\hline & \multicolumn{3}{|c|}{ Temperature } & \multicolumn{3}{c|}{ Blood Pressure } & \multicolumn{3}{c|}{ Heart Beat } \\
\cline { 2 - 10 } Patients & Low & Normal & High & Low & Normal & High & Low & Normal & High \\
\hline Patient1 & 61 & 91 & 92 & 126 & 165 & 166 & 69 & 81 & 91 \\
\hline Patient 2 & 63 & 89 & 93 & 127 & 167 & 170 & 70 & 80 & 93 \\
\hline Patient 3 & 66 & 90 & 91 & 130 & 170 & 171 & 71 & 82 & 90 \\
\hline Patient 4 & 64 & 92 & 91 & 126 & 167 & 170 & 70 & 84 & 92 \\
\hline Patient 5 & 64 & 92 & 91 & 126 & 167 & 170 & 70 & 84 & 91 \\
\hline
\end{tabular}

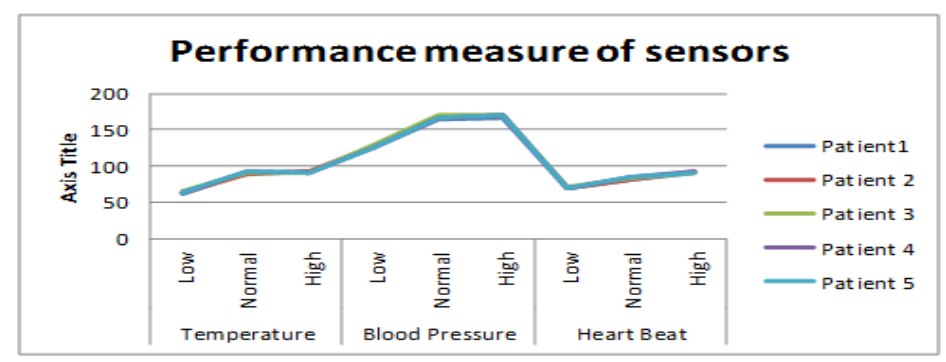

Figure 3. Performance measure of different Sensor

The Figure 4 depicts the evidence that on 10 patients (c1-c10) tests were performed with two set of groups satisfied and non-satisfied. This AMM application performance level of efficiency reached $90 \%$.

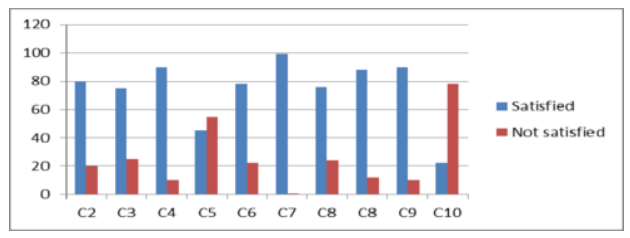

Figure 4. Performance of AMM 


\section{Conclusion}

In this paper, we proposed an Anytime Medical Machine (AMM). This is based on temperature sensor, an ultrasonic sensor, and a heartbeat and local cell sensor. The proposed application was developed with windows operating system using Java language, MySQL database, IDE Net beans and cloud storage as Drop box. Based on our outputs, we can conclude that the AMM application can be an online tool that supports people as effective telemedicine. The outputs with 10 patients produced good result. Thus outputs confirm the efficiency and performance of the application.In Future, the hospital nearest can be found for non-solvable causes. The AMM set up helps GPS in tracking the nearest hospitals and calling the ambulance for patient in the case of emergency. Making cloud storage completely for proper way of maintaining the patient information. Maintenance of patient information is possible. The time taken by the patient taking the medicine and monitoring patient information through mobile phone can be assessed using their government ID.

\section{References}

[1] Saravanan. M., and Achsah Mary Marks, MEDIBOX-IoT Enabled Patient Assisting Device, IEEE 4th World Forum on Internet of Things (WF-IoT), 2018.

[2] Amutha, B., Manickavasagam, B., Alankrit Patnaik and KarthickNanmaran, Erection of Comprehensive Wellness Programme for Global Healthcare Monitoring System using AODV Protocol with Data Clustering Schema, Indian Journal of Science and Technology, 2015, 8(17) ,1-13.

[3] Eric Johnson,M., and AjitAppari, Information Security and Privacy in Healthcare: Current State of Research, International Journal of Internet and Enterprise Management, 2010, 6(4), 279 - 314.

[4] Chih-Ming Chen, Chin-Ming Hong, Chia-Meng, Huang and Tai-Hung Lee, Web-based Remote Human Pulse Monitoring Systemwith Intelligent Data Analysis for Home Healthcare, Expert Systems with Applications, 2011, 38, 3, 2011-2019.

[5] Bhagya, S.L., and Raju K. Gopal., A novel data storage solution for cloud, International Conference on Networks \& Advances in Computational Technologies (NetACT), IEEE, 2017.

[6] Sarah Shaikh and DeepaliVora, Secure cloud auditing over encrypted data, International Conference on Communication and Electronics Systems (ICCES), IEEE, 2016.

[7] Wilson G. de Oliveira Junior et. al., A proposal for Internet of Smart Home Things based On BCI system to aid patients with amyotrophic lateral sclerosis, Neural Computing and Applications, Springer, 2018.

[8] Mary Livinsa.Z, Mary Valantina,G., E Health Monitoring Systems in Smart Environments, J. Pharm. Sci. \& Res. Vol. 11(9), 2019, 3130-3132.

[9] S.Hema Kumar, J.Uday Kiran, V.D.Ambeth Kumar, G.Saranya, Ramalakshmi V.Effective Online Medical Appointment System. International Journal of Scientific \& Technology Research.Volume 8.Issue 09, September 2019. p $803-.805$. 